Varia - hiver 2020

\title{
Effets de la formation et de l'accompagnement pédagogiques sur le niveau de centration sur l'apprentissage des nouveaux professeurs
}

Louise Ménard, Christian Hoffmann, Sébastien Boucher and Martin Riopel

\section{OpenEdition}

Journals

Electronic version

URL: http://journals.openedition.org/ripes/2527

DOI: $10.4000 /$ ripes.2527

ISSN: 2076-8427

Publisher

Association internationale de pédagogie universitaire

\section{Electronic reference}

Louise Ménard, Christian Hoffmann, Sébastien Boucher and Martin Riopel, « Effets de la formation et de l'accompagnement pédagogiques sur le niveau de centration sur l'apprentissage des nouveaux professeurs », Revue internationale de pédagogie de l'enseignement supérieur [Online], 36(1) | 2020, Online since 11 April 2020, connection on 08 September 2020. URL : http://journals.openedition.org/ ripes/2527 ; DOI : https://doi.org/10.4000/ripes.2527

This text was automatically generated on 8 September 2020

Article L.111-1 du Code de la propriété intellectuelle. 


\title{
Effets de la formation et de
} l'accompagnement pédagogiques sur le niveau de centration sur l'apprentissage des nouveaux professeurs

\author{
Louise Ménard, Christian Hoffmann, Sébastien Boucher and Martin Riopel
}

\section{Introduction}

1 À l'heure où la réussite au premier cycle universitaire est devenue une réelle préoccupation, les universités canadiennes et françaises ont décidé de mettre en place des services de pédagogie favorisant le développement d'un enseignement plus centré sur les étudiants (Bertrand, 2014; Conseil supérieur de l'éducation, 2013; Raby, 2011). Les nouveaux professeurs (désigne aussi les maîtres de conférence) au Canada et en France constituent une des clientèles cibles de ces services ${ }^{1}$, car à l'enseignement supérieur ils ne sont pas préparés à enseigner et tendent à se centrer sur la transmission d'un contenu disciplinaire (Langevin, Grandtner et Ménard, 2008; Ménard, 2012; Romainville et Michaud, 2012). Mais les formations courtes et l'accompagnement pédagogiques qu'on offre généralement (Duguet, Lambert Le Mener, Morlaix, 2016; Frenay et al., 2010) favorisent-ils le développement d'une pratique plus centrée sur l'apprentissage étudiant chez les nouveaux professeurs? Question pertinente à laquelle de nombreux chercheurs s'intéressent depuis le milieu des années 1960.

2 L'article de Stes, Min-Leliveld, Gijbels et Van Petegem (2010) constitue une source intéressante, car les auteurs analysent 36 recherches portant sur les effets de différents programmes de développement pédagogique sur les professeurs. Il en ressort les éléments suivants : 1) les formations se déployant sur une longue durée ont un plus grand impact sur les pratiques que celles qui ont lieu une seule fois; 2) d'autres 
dispositifs comme l'accompagnement ou le tutorat par les pairs ont plus d'impact sur les pratiques que la formation et 3) les dispositifs regroupant des professeurs d'une ou de plusieurs disciplines ont des effets comparables.

3 Les autres recherches répertoriées se sont intéressées à la portée d'un seul dispositif. Ainsi des recherches réalisées dans quatres pays différents se sont centrées sur les effets de la formation pédagogique sur les conceptions et les approches des professeurs (Hanbury, Prosser et Rickinson, 2008; Postareff, Lindblom-Ylänne et Nevgi, 2008; Stes et Van Petegem, 2011; Trigwell, Caballero Rodriguez et Han, 2012). Elles établissent un lien positif entre la formation longue et le passage d'une approche centrée sur la transmission de connaissances à une approche davantage centrée sur l'apprentissage étudiant. Une recherche plus récente, cette fois française (Bailly, Demougeot-Lebel et Lison, 2015), a examiné les effets d'une formation courte de 18 heures offerte à 36 professeurs nouvellement embauchés. Les auteurs constatent que les professeurs qui ont majoritairement rapporté une évolution de leurs conceptions de l'enseignement disent, dans une proportion de seulement $58 \%$, avoir mis en place de nouvelles pratiques. Kiffer (2016) affiche des résultats différents en ce qui concerne les effets sur les conceptions des nouveaux professeurs français. Il explique que parmi les 376 ayant répondu à un questionnaire répertoriant les dispositifs auxquels les nouveaux professeurs recourent pour développer leurs compétences pédagogiques, $44 \%$ notent avoir suivi une ou des formations pédagogiques, mais seulement $8 \%$ estiment que la ou les formations ont entrainé une remise en question de leurs conceptions de l'enseignement.

4 L'accompagnement pédagogique a également fait l'objet de plusieurs recherches à l'enseignement supérieur, la plupart anglo-saxonnes. Rivers (2005), suite à une recension des écrits, conclut que l'accompagnement individualisé favorise le développement de pratiques plus centrées sur l'apprentissage lorsque le conseiller pédagogique s'appuie sur le vécu des professeurs plutôt que de se limiter à fournir de l'information sur les bonnes pratiques. Finellli et al. (2008) rapportent aussi de nombreuses études démontrant que l'accompagnement peut avoir un effet positif sur l'enseignement. Les écrits recensés par ces chercheurs basent fréquemment leurs conclusions sur les notes obtenues par les étudiants ou leurs évaluations de l'enseignement. Au Canada et en France, l'accompagnement semble toutefois un dispositif beaucoup moins souvent utilisé que dans les pays anglo-saxons. Pour exemple, Kiffer (2016) rapporte que $10 \%$ de nouveaux professeurs ayant répondu au questionnaire mentionnent avoir consulté un conseiller pour une aide pédagogique, généralement une ou deux fois l'an.

5 Ainsi, aussi bien la recension de Stes et al. (2010) que les autres travaux portant sur les effets de la formation et de l'accompagnement à l'université démontrent que les chercheurs recourent généralement à des données autorapportées pour en établir les effets. De plus, nous constatons que les recherches du côté francophone demeurent rares (Daele et Sylvestre, 2016).

6 Étant donné que les universités privilégient la formation de courte durée ou l'accompagnement des nouveaux professeurs pour développer un enseignement plus centré sur l'apprentissage étudiant, il apparaît pertinent d'étudier les effets de chaque dispositif et de leur combinaison en s'appuyant sur l'observation des pratiques au fil du temps plutôt que de se limiter aux réponses fournies à une seule occasion ou peu après l'intervention. Il semble également approprié de se demander si les professeurs selon 
un contexte d'exercice différent se comportent différemment. Pour y arriver, nous avons privilégié une étude ${ }^{2}$ 'une durée de trois ans auprès de professeurs canadiens et français. Au terme de cette recherche, nous nous sommes également intéressés aux perceptions que les professeurs ont de leurs pratiques pour comprendre de quelle manière ils voient leur développement. Finalement, nous avons cherché à identifier les autres ressources qu'ils ont consultées dans le but de développer lesdites pratiques d'enseignement.

7 Dans le cadre de cet article, sont d'abord abordés le développement pédagogique puis les dispositifs étudiés. Ensuite, sont définies les pratiques d'enseignement que nous comptons observer, en précisant ce qu'implique le niveau de centration sur l'apprentissage. Dans la section méthodologie, sont détaillés l'outil d'observation, le déroulement de l'observation effectuée auprès de nouveaux professeurs qui ont été uniquement accompagnés ou formés, qui ont été formés puis accompagnés et qui n'ont été ni formés ni accompagnés ainsi que la brève interview réalisée à la fin de la recherche. L'article présente finalement les résultats en décrivant les effets sur les pratiques observées ainsi que les autres stratégies de développement pédagogique utilisées, puis les analyse en répondant aux objectifs de la recherche.

\section{Cadre théorique}

8 Nous définissons d'abord le concept de développement pédagogique et puis nous abordons la formation et l'accompagnement offerts pas les services de pédagogie pour le soutenir. Ensuite, nous décrivons les pratiques d'enseignement et précisons l'approche cognitive ${ }^{3}$ à partir de laquelle est définie la centration sur l'apprentissage.

\subsection{Le développement pédagogique des nouveaux professeurs}

9 Le développement pédagogique, selon Frenay, Jorro et Poumay (2011), constitue un champ de recherche et de pratiques «qui regroupe les écrits sur les dispositifs et les interventions favorisant le développement des compétences pédagogiques des professeurs de l'enseignement supérieur, sans toutefois analyser le processus même de développement de ces compétences pédagogiques» (p.106). Étant donné que cette étude porte sur les effets de la formation et de l'accompagnement sur les pratiques d'enseignement des professeurs, il est évident que nous nous intéressons au développement pédagogique des professeurs tel que défini par Frenay et al. (2011).

C'est en cohérence avec cette définition que Frenay a réfléchi avec une équipe de chercheurs à l'élaboration d'un cadre de référence permettant de conceptualiser la pratique et la recherche sur le développement pédagogique. La perspective retenue par cette équipe "se centre sur le développement des capacités d'enseignement et d'apprentissage des professeurs » (Frenay et al., 2010, p. 66). Elle fait, dès lors, appel à tout ce qui peut être fait pour aider les professeurs à apprendre à enseigner de manière à soutenir l'apprentissage des étudiants. Ce qui implique que le professeur prenne le temps de réfléchir à son développement pédagogique et que l'université fournisse « des conditions qui leur permettent de reconnaitre les besoins de leurs étudiants, d'acquérir des connaissances et des compétences en matière d'enseignement et d'apprentissage, de partager celles-ci avec leurs collègues, d'être reconnus et valorisés pour leur travail.» (Frenay et al., 2010, p. 66). Cette conceptualisation de la pratique et de la 
recherche ne peut se soustraire au fait que les approches et les pratiques de chaque université s'inscrivent dans un contexte national et une politique qui lui sont propres, dans une institution et dans un environnement disciplinaire (Beguin, 2007; Dall'Alba et Sandberg, 2006). Les pratiques observées des professeurs seront donc situées dans un contexte propre à un pays, une université, une discipline, etc. qui fournit des ressources, mais qui impose aussi des contraintes qui ont un effet sur leur développement.

11 Pour favoriser le développement pédagogique des professeurs, les universités ont placé en première ligne des conseillers pédagogiques qui ont pour mandat de former, accompagner, conseiller les professeurs dans le développement de leurs compétences pédagogiques, leur habilité à soutenir l'apprentissage (Taylor et Rege Colet, 2010). Différents dispositifs sont offerts aux professeurs pour favoriser leur développement comme la formation courte et longue abordant l'ensemble des tâches du professeur, les ateliers sur des sujets ciblés, l'accompagnement d'un professeur ou d'un groupe de professeurs. Nous aborderons dans cette recherche les deux dispositifs les plus fréquemment mobilisés: la formation courte et l'accompagnement individuel.

12 La formation constitue la voie privilégiée pour faire évoluer les conceptions et les pratiques des professeurs de manière à ce qu'ils se centrent davantage sur l'apprentissage étudiant (Luzeckyj et Badger, 2008). Cette formation, qui s'appuie sur les principes de l'approche cognitive, soutient que le rôle de l'enseignant est désormais de concevoir et d'animer des activités d'apprentissage dans lesquelles les étudiants mobilisent leurs connaissances pour faire face à des situations nouvelles et motivantes qui les amènent à interagir pour chercher et traiter l'information (Annoot, 2014).

Quant à l'accompagnement, il est défini par plusieurs auteurs comme une activité de consultations individualisée visant à soutenir le développement pédagogique des professeurs (Clement, Di Napoli, Annelies Gilis, Buelens, et Frenay, 2011). Kozanitis, Demougeot-Lebel et Daele (2016) préciseront qu'il a pour finalité « l'amélioration de la qualité de l'expérience d'apprentissage de l'étudiant.» (p.24). Les consultations que font les conseillers pédagogiques portent généralement sur les dimensions pratiques de l'enseignement. Une démarche d'accompagnement implique souvent plusieurs rencontres.

14 Arpin et Capra (2008) considèrent que l'accompagnement est important, car il leur permet « d'amorcer ou de poursuivre des changements, de modifier leur manière de faire, et de les rendre conscients de leurs gestes pédagogiques.» (p. IIV). Pour y parvenir, l'accompagnateur offre une " présence attentionnée » (Paul, 2015, p. 25) dont le but est d'aller où l'autre va, à son rythme et à sa mesure, sans décider ou faire à sa place et en prenant en considération le contexte dans lequel il se trouve. Il adopte alors " une posture d'écoute facilitant le questionnement réflexif d'une personne sur ce qu'elle veut et peut dans une situation dans laquelle elle est prise et partie prenante. » (Paul, 2015, p. 22). L'accompagnement, selon Biemar (2012), implique également que l'accompagnateur soit en mesure de négocier les rôles et les actions de chacun et de soutenir concrètement l'accompagné en visant in fine son autonomie. 


\subsection{L'observation des pratiques d'enseignement centrées sur l'apprentissage} professeurs. Cet intérêt est lié à la nécessité d'améliorer la compréhension de ce qui affecte la qualité des processus d'enseignement et les résultats d'apprentissage (Lenoir, 2012). Ce que nous entendons ici par pratiquées observées correspond davantage à ce que Clanet et Talbot (2012) nomment des pratiques constatées dans le sens où ces pratiques résultent d'observation des pratiques ou des activités en contexte réel d'enseignement.

Comme mentionné, les dispositifs visent le développement de pratiques d'enseignement s'appuyant sur l'approche cognitive. Cette approche met l'accent sur les processus actifs du traitement d'information et présente l'apprentissage comme une série de transformations de la connaissance qui s'opèrent à travers les structures du cerveau et qui entraînent une modification de la structure cognitive (Rocheleau, 2009). Pour enseigner selon cette approche, le professeur procède à l'analyse des apprentissages à réaliser pour cerner les objectifs à atteindre. Ensuite, il voit à ce que l'étudiant donne du sens et il favorise un traitement actif des nouvelles connaissances en considérant les connaissances antérieures et en provoquant le conflit cognitif. Il intervient afin d'assurer l'organisation hiérarchique et la contextualisation desdites connaissances dans la mémoire et il soutient chez l'étudiant une démarche cognitive et métacognitive de manière à consolider les apprentissages (Parmentier et Paquay, 2002; Tardif, 1997). Il prévoit également des activités d'application et de transfert. Pour les rendre possible, le professeur met en place des activités de résolution de problèmes dans des contextes plus complexes que ceux ayant servi d'appui à l'enseignement (Frenay et Bédard, 2004; Tardif, 1999).

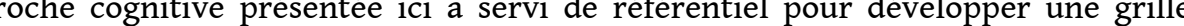
d'observation des pratiques en cours et pour estimer leur degré de centration sur l'apprentissage. D'emblée, il faut préciser que cette grille s'inspire fortement de celle proposée par Saint-Pierre, Bédard et Lefebvre $(2012,2014)$ suite à une recherche qui avait pour objectif d'étudier en profondeur les pratiques d'enseignement de dix-huit professeurs qui interviennent dans des programmes utilisant des pédagogies ayant pour but une plus grande centration sur l'apprentissage. La dite grille comporte sept catégories fondées sur un cadre de référence cognitiviste : 1 ) agir sur les connaissances antérieures, 2) soutenir l'activité intellectuelle, 3) susciter et exploiter les interactions, 4) soutenir l'organisation des connaissances, 5) intégrer l'évaluation dans les situations d'apprentissage, 6) favoriser le transfert, 7) développer la capacité réflexive. Ces catégories sont déclinées selon trois niveaux de centration sur l'apprentissage :

- Niveau $1:$ l'enseignant effectue les opérations cognitives et métacognitives et les étudiants jouent un rôle plutôt passif.

- Niveau 2: l'enseignant incite les étudiants à effectuer certaines opérations cognitives et métacognitives. Il pose des questions pour les amener à effectuer des activités simples.

- Niveau $3:$ l'enseignant incite les étudiants à décider et à effectuer des opérations cognitives et métacognitives. Il sollicite la réalisation d'activités variées et complexes.

Ces niveaux ont été définis en fonction de la complexité des opérations cognitives et métacognitives. Cette déclinaison des trois niveaux de centration sur l'apprentissage a été conservée, car elle permettait de voir si le niveau évoluait au fil du temps. Par 
ailleurs, deux catégories de pratiques ont été ajoutées à cette grille toujours avec trois niveaux de centration afin qu'elle soit en cohérence avec l'ensemble des principes de l'approche cognitive: 8) donner du sens à l'apprentissage et 9) accompagner les étudiants dans la réalisation de leurs activités.

\subsection{Objectifs}

Étant donné que la recherche vise à cerner les effets possibles d'une formation courte ou de l'accompagnement des nouveaux professeurs canadiens et français en les comparant à des professeurs non formés non accompagnés et en tenant compte du contexte et des autres possibilités de développement pédagogique, nous retenons les objectifs suivants: 1) déterminer le niveau de centration sur l'apprentissage des pratiques d'enseignement des nouveaux professeurs formés et accompagnés, formés ou accompagnés et non formés non accompagnés canadiens et français au cours de trois années consécutives; 2) décrire les perceptions de chacun à l'égard de ses pratiques au terme de la recherche; 3 ) circonscrire les autres ressources utilisées par ces professeurs pour développer leurs pratiques d'enseignement et en préciser l'utilité.

\section{Méthodologie}

20 Cette section décrit les sujets, les outils de collecte qui ont permis d'accéder aux pratiques des professeurs et à leur discours, puis le déroulement de la collecte et les modalités d'analyse.

\subsection{Sujets}

21 Le recrutement des participants a été effectué à partir des listes de nouveaux professeurs fournies par les universités concernées. Les professeurs intéressés ont été rencontrés individuellement par la responsable de la recherche ou par un des assistants de recherche. Les assistants de recherche sont des étudiants de second ou troisième cycles que nous avons formés et encadrés et qui ont été impliqués dans toutes les étapes de la recherche. Au moment de la rencontre, les professeurs qui souhaitaient s'inscrire à la formation, ceux qui voulaient également ou uniquement être accompagnés par un conseiller pédagogique et ceux qui ne souhaitaient ni l'un ni l'autre étaient répertoriés. Les participants étaient également informés des règles éthiques concernant l'anonymat et de la confidentialité.

22 Au départ, 51 nouveaux professeurs ont répondu vouloir participer à la recherche. Parmi ceux-ci, seulement quelques-uns ont abandonné en cours de recherche. D'autres n'ont pas été retenus pour cette analyse, car ils n'ont pas donné le même cours durant les trois années. Nous avons fait le choix de ne pas les inclure, car il paraissait important de contrôler la variable "séance de cours identique " dans un contexte chargé de dissemblances comme toutes les recherches basées sur le volontariat. Malheureusement, le nombre de professeurs ayant accepté de participer à la recherche étant restreint, nous n'avons pas pu choisir les participants en prenant en compte d'autres variables.

23 Cette recherche a regroupé 34 professeurs de 2 universités francophones canadiennes et de 3 universités françaises appartenant à des disciplines diverses (Tableau 1) et ayant 
tous moins de 3 ans d'expérience en tant que professeurs d'université au début de la recherche. Parmi ces 34 nouveaux professeurs, 2 Canadiens et 7 Français n'ont choisi ni formation ni accompagnement, 5 Canadiens et 3 Français se sont inscrits à la formation jumelée à un accompagnement, 7 Canadiens et 3 Français se sont inscrits volontairement à une courte formation à l'enseignement, 2 Canadiens et 5 Français ont été uniquement accompagnés. Les professeurs canadiens ont été observés durant une même séance de cours de trois ou quatre heures et les professeurs français durant une même séance d'un module ou d'une unité d'enseignement d'une heure et demi ou deux heures, et ce durant trois années consécutives, c'est-à-dire à trois reprises. Pour protéger l'anonymat de chaque professeur, l'assistant devait lui attribuer un code permettant de repérer à quel pays (CA pour les Canadiens et FR pour les Français), à quelle université (A, B, C pour les universités françaises et D, E pour les canadiennes) et à quel regroupement (par exemple, 10 pour les non formés non accompagnés) il appartenait.

Tableau 1: Disciplines enseignées des quatre regroupements de professeurs

\begin{tabular}{|c|c|c|c|c|c|c|c|c|}
\hline \multirow[t]{2}{*}{$\begin{array}{l}\text { Domaines } \\
\mathrm{N}=34\end{array}$} & \multicolumn{2}{|c|}{$\begin{array}{l}\text { Non formés } \\
\text { non acc } \\
(\mathrm{N}=9)\end{array}$} & \multicolumn{2}{|c|}{$\begin{array}{l}\text { Accompagnés } \\
(\mathrm{N}=7)\end{array}$} & \multicolumn{2}{|l|}{$\begin{array}{l}\text { Formés } \\
(\mathrm{N}=10)\end{array}$} & \multicolumn{2}{|c|}{$\begin{array}{l}\text { Formés acc } \\
(\mathrm{N}=8)\end{array}$} \\
\hline & Canada & France & Canada & France & Canada & France & Canada & France \\
\hline $\begin{array}{l}\text { Droit/science politique } \\
(\mathrm{N}=6)\end{array}$ & & $\begin{array}{l}\text { FR- } \\
\text { A10 } \\
\text { FR- } \\
\text { A13 }\end{array}$ & & & $\begin{array}{l}\text { CA- } \\
\text { D52 } \\
\text { CA- } \\
\text { D53 }\end{array}$ & $\begin{array}{l}\text { FR- } \\
\text { A50 }\end{array}$ & CA-E33 & \\
\hline $\begin{array}{l}\text { Éducation/ } \\
\text { psychologie }(\mathrm{N}=3)\end{array}$ & $\begin{array}{l}\text { CA- } \\
\text { D10 }\end{array}$ & & $\begin{array}{l}\text { CA- } \\
\text { D20 }\end{array}$ & & & & CA-E34 & \\
\hline $\begin{array}{l}\text { Sciences et technologie } \\
(\mathrm{N}=8)\end{array}$ & & $\begin{array}{l}\text { FR-B10 } \\
\text { FR-B11 }\end{array}$ & & $\begin{array}{l}\text { FR-B20 } \\
\text { FR-B21 } \\
\text { FR-B22 } \\
\text { FR- } \\
\text { A20 }\end{array}$ & $\begin{array}{l}\text { CA- } \\
\text { D56 }\end{array}$ & & & $\begin{array}{l}\text { FR- } \\
\text { A32 }\end{array}$ \\
\hline $\begin{array}{l}\text { Arts et lettres } \\
(\mathrm{N}=6)\end{array}$ & & $\begin{array}{l}\text { FR- } \\
\text { A14 }\end{array}$ & $\begin{array}{l}\mathrm{CA}- \\
\mathrm{D} 21\end{array}$ & & $\begin{array}{l}\text { CA- } \\
\text { D51 } \\
\text { CA- } \\
\text { D50 } \\
\text { CA- } \\
\text { D54 } \\
\text { CA- } \\
\text { D55 }\end{array}$ & & & \\
\hline
\end{tabular}




\begin{tabular}{|c|c|c|c|c|c|c|}
\hline $\begin{array}{l}\text { Sciences de la santé } \\
(\mathrm{N}=5)\end{array}$ & & & & $\begin{array}{l}\text { FR-C50 } \\
\text { FR-C51 }\end{array}$ & CA-E32 & $\begin{array}{l}\text { FR- } \\
\text { A30 } \\
\text { FR- } \\
\text { A31 }\end{array}$ \\
\hline $\begin{array}{l}\text { Sciences humaines/ } \\
\text { communication } \\
(\mathrm{N}=5)\end{array}$ & & $\begin{array}{l}\text { FR- } \\
\text { A11 } \\
\text { FR- } \\
\text { A12 }\end{array}$ & $\begin{array}{l}\text { FR- } \\
\text { A21 }\end{array}$ & & $\begin{array}{l}\text { CA-E30 } \\
\text { CA-E31 }\end{array}$ & \\
\hline $\begin{array}{l}\text { Gestion/économie } \\
(\mathrm{N}=1)\end{array}$ & $\begin{array}{l}\text { CA- } \\
\text { D11 }\end{array}$ & & & & & \\
\hline
\end{tabular}

\subsection{Formation}

Plusieurs échanges ont eu lieu entre l'équipe de recherche et les conseillers pédagogiques qui dispensent la formation afin d'assurer une offre semblable s'inscrivant dans une approche cognitive. Il a été décidé que la formation aborderait les thèmes suivants : la définition des objectifs d'apprentissage, la nécessité de faire des liens avec les connaissances antérieures, de donner du sens à l'apprentissage, d'utiliser des stratégies actives d'enseignement, de planifier l'application et le transfert des apprentissages, de prendre en compte les processus cognitifs de l'apprentissage, de bien choisir les modalités, les outils et les critères d'évaluation et de fournir des rétroactions. Elle avait pour but d'amener les professeurs à réfléchir aux effets de leurs pratiques sur l'apprentissage et à s'engager dans un processus de développement pédagogique leur permettant de s'adapter et de s'améliorer (Stes et Van Petegem, 2011).

$\mathrm{Au}$ tout début de la formation, les conseillers pédagogiques ont présenté le cadre théorique choisi, c'est-à-dire l'approche cognitive, puis chaque thème de la formation a été explicité. À chaque fois qu'ils ont abordé un thème, un échange avec les professeurs a été amorcé au sujet de leurs conceptions et de leurs pratiques actuelles. De nombreuses mises en application ont été proposées afin de favoriser une certaine intégration des apprentissages.

L'offre de formation a répondu aux contraintes horaires imposées par les universités participantes, c'est-à-dire qu'elle a été de 15 à 18 heures. Elle a eu lieu deux semaines avant ou au début des cours de la session d'automne 2014.

\subsection{Accompagnement}

L'équipe de recherche et les conseillers pédagogiques ont également travaillé ensemble afin de déterminer les étapes de la démarche d'accompagnement. Cette démarche s'inspire des perspectives d'Arpin et Capra (2008), de Paul (2015), de Biemar (2012) et du cycle de l'apprentissage expérientiel de Kolb (1984). Les étapes déterminées ont été les suivantes : 1) accueillir le professeur, établir un climat de confiance et l'amener à décrire sa situation, ses besoins; 2) analyser la situation, le problème avec lui; 3) l'aider à mettre en perspective ses acquis (formation, lectures, etc.); 4) identifier avec lui des 
pistes de solution réalistes; 5) cerner les apprentissages à faire ou les compétences à développer et 6) assurer un suivi pédagogique d'une rencontre à l'autre. Les conseillers pédagogiques avaient explicitement pour rôle d'amener le professeur à réfléchir sur sa pratique puis de l'aider à préciser et à réaliser les changements souhaités en fournissant, au besoin, du conseil ou les ressources nécessaires.

Les professeurs qui souhaitaient être accompagnés ont été informés qu'ils s'engageaient à rencontrer un ou une conseillère pédagogique à trois reprises chaque année. Les professeurs ont finalement été rencontrés entre 4 et 6 fois durant la période d'accompagnement, à l'exception d'un professeur français (FR-B21) rencontré 2 fois.

\subsection{Grille d'observation}

La grille d'observation (Annexe 1) a été validée par les assistants de recherche qui l'ont utilisée auprès de huit professeurs canadiens et français. Une analyse des difficultés rencontrées au moment de l'observation a ensuite été faite par les assistants et l'équipe de recherche. Certains items ont été reformulés pour être compris de la même manière par tous.

\subsection{Collecte et analyse de données}

La collecte de données auprès de chaque professeur a été réalisée par un assistant de recherche qui a été formé à faire de l'observation directe et à utiliser la grille. Les pratiques ont été observées à la sixième semaine de la session, afin de laisser le temps au professeur de s'adapter à ses étudiants. La même séance a été observée durant les trois ans de la recherche.

Le niveau de centration étant détaillé pour chaque pratique appartenant à chaque catégorie, il a été relativement simple de noter le niveau observé. Quant à la fréquence, elle a été répertoriée de la manière suivante : jamais lorsque la pratique n'était pas observée, parfois lorsqu'elle était observée d'une à quatre fois et souvent, lorsqu'elle était observée à plus de quatre reprises ou dans le cadre d'activités de longue durée. Pour analyser le grand nombre de données récoltées, nous avons traduit les observations consignées dans les grilles par des scores chiffrés.

Pour faciliter la lecture et l'analyse des résultats, les scores ont été calculés par professeur ou par regroupement de professeurs et avec différents degrés d'agrégation. Nous présentons les résultats sous cette forme afin de rendre comparables les neuf catégories de pratiques et les trois niveaux de centration entre eux.

Pour coder la fréquence de chaque pratique observée, les chiffres suivants ont été attribués (code fréquence) : «jamais » $=0$; «parfois » $=1$; « souvent $=2$. Dans un premier temps, un score normalisé pour chaque catégorie de pratique et chaque niveau de centration sur l'apprentissage a été calculé de la façon suivante : les codes fréquence de tous les items d'un même niveau de centration ont été additionné, puis divisé par le score maximal possible. Ainsi, un score normalisé de 0 signifie que tous les items relatifs à une catégorie de pratiques et au niveau concernés sont cochés « jamais » alors que 1 veut dire que tous les items sont cochés «souvent». Aux fins de l'analyse, ces données ont ensuite été agrégées de deux façons, la première donnant des résultats pour les regroupements des professeurs, la deuxième pour chaque professeur individuellement. Pour ce faire, nous avons effectué les calculs suivants : 
34 a) calcul de la moyenne des scores normalisés pour chaque catégorie de pratiques, par an et par niveau, pour tous les professeurs d'un même regroupement, ce qui permet de comparer l'évolution des différentes catégories de pratiques entre les quatre regroupements;

b) calcul de la moyenne des scores a. sur les neuf pratiques ce qui permet de comparer l'évolution "globale» de la centration sur l'apprentissage étudiant des quatre regroupements de professeurs;

c) calcul pour chaque professeur d'un indicateur unique par an, appelé «centration »; ce calcul se fait en deux étapes :

1. pour chaque niveau de centration, calcul de la moyenne des scores normalisés sur les neuf catégories de pratiques;

2. addition pondérée des scores de chaque niveau : centration = score au niveau $1+2 \times$ (score au niveau 2$)+3 \mathrm{x}$ (score au niveau 3$)$.

La somme pondérée accorde plus de poids aux pratiques des niveaux 2 et 3 (degrés de centration plus forts), car une pratique au niveau 2 se traduit par des activités étudiantes qui prennent généralement plus de temps qu'au niveau 1 et c'est d'autant plus vrai au niveau 3. Cet indicateur " centration", qui peut possiblement varier entre 0 et 6, permet d'étudier l'évolution globale des pratiques de chaque professeur.

Pour avoir accès aux perceptions des professeurs concernant leurs pratiques et connaître les autres ressources utilisées pour les développer, nous avons posé les questions suivantes: quel bilan faites-vous de vos pratiques d'enseignement au terme de ces trois années? Avez-vous cherché, au cours de la recherche, à vous former ou à obtenir des informations pour vous aider dans votre enseignement? Avez-vous, par exemple, suivi une formation thématique ou participé à un atelier d'une ou deux heures, rencontré un conseiller ou une conseillère en pédagogie, consulté des ouvrages sur la pédagogie, échangé avec vos pairs? Les réponses fournies ont été catégorisées en fonction des bilans jugés positifs, plus ou moins positifs ou négatifs et des sources de formation ou d'information identifiées.

\section{Résultats}

La section présente une synthèse des pratiques centrées sur l'apprentissage des professeurs canadiens et français, le bilan qu'ils font de leurs pratiques ainsi qu'une identification des autres sources de formation qui, de leur point de vue, ont influencé leurs pratiques.

\subsection{Pratiques observées}

Dans un premier temps, regardons l'indicateur "centration" pour chacun des 34 professeurs en identifiant leur discipline respective. Dans l'annexe 2, les niveaux de centration sont affichés pour l'an 1 et l'an 3 ainsi que leur développement résultant de la différence entre l'an 3 et l'an 1 . Ils sont présentés sous la forme d'un tableau pour chaque regroupement.

41 D'emblée, nous observons une forte variation du niveau de centration des professeurs de chaque regroupement à l'an 1. L'écart entre la centration la plus faible et la plus forte varie entre 2 et 3 , avec toutefois des moyennes comparables entre les quatre 
regroupements (entre 1,37 et 2). Les résultats obtenus pour l'évolution entre la première et la troisième observations suggèrent de distinguer trois cas : des pratiques en nette régression (évolution entre $-1,76$ et $-0,75$ ) pour 8 professeurs; peu d'évolution de la centration (entre $-0,37$ et 0,31 ) pour 18 professeurs; des pratiques en nette progression (entre 0,66 et 1,32 ) pour 8 professeurs. Il faut noter que dans chacun des quatre regroupements, on trouve des professeurs dans les trois cas: "régression notable » (entre 1 et 3 ), «peu ou pas d'évolution » (entre 3 et 5 ) et «progrès notable » (entre 1 et 3), mais que la majorité des professeurs (18 sur 34) évolue peu ou pas. Ces résultats ne semblent pas liés à la discipline enseignée, car les professeurs concernés sont de disciplines variées. Si nous regardons maintenant ceux qui progressent et régressent, il est à remarquer que parmi les professeurs qui régressent, six sur huit sont Français. Quant à ceux qui affichent un progrès notable, on compte cinq professeurs canadiens et trois professeurs français. Il semble que les professeurs canadiens maintiennent ou font évoluer les trois niveaux de centration sur l'apprentissage en plus grand nombre que les professeurs français.

Dans un deuxième temps, nous avons étudié les neuf catégories séparément. Dans les tableaux de l'annexe 3, les résultats pour la moyenne des scores normalisés sont présentés pour chaque catégorie de pratiques, par an et par niveau, pour tous les professeurs d'un même regroupement. Pas de tendances lourdes, si ce n'est que la catégorie « Susciter et exploiter les interactions » progresse systématiquement sur les 3 niveaux de centration entre l'an 1 et l'an 3 et indépendamment du regroupement, alors que pour la catégorie «Donner sens à l'apprentissage ", c'est l'inverse pour la grande majorité.

Finalement, nous avons comparé les professeurs canadiens et français dans l'évolution de leurs pratiques. Les tableaux de l'annexe 4 présentent les mêmes résultats que les tableaux de l'annexe 3, mais cette fois la moyenne est faite sur tous les professeurs d'un même pays. Nous constatons que les résultats pour les trois niveaux sont quasiment toujours plus élevés pour les professeurs français. En effet, les moyennes sur les 9 catégories de pratiques pour les canadiens sont à 0,33 au niveau 1, 0,28 au niveau 2 et 0,12 au niveau 3 alors que pour les Français, elles sont à 0,55 au niveau 1, 0,43 au niveau 2 et 0,20 au niveau 3 au début de l'étude. La différence est remarquable pour « Donner du sens à l'apprentissage » dont les scores sont beaucoup plus élevés pour les Français que pour les Canadiens. Par ailleurs, en ce qui concerne l'évolution (an 3 - an 1) des professeurs, les Canadiens ont tendance à progresser (4 niveaux sont en régression sur les 25 scores en total), alors que la tendance est inversée pour les Français (6 niveaux en progression sur 25 scores) Nous faisons le même constat lorsque nous analysons les résultats de l'annexe 2 .

\subsection{Bilan des pratiques d'enseignement}

$\mathrm{Au}$ terme de la recherche, les professeurs ont été invités à faire un bilan de leurs pratiques d'enseignement. Dans les tableaux présentés à l'annexe 2 , se retrouvent l'orientation du bilan effectuée par chacun (positif (+), négatif $(-)$ ou plus ou moins positif (+-)) et un résumé de l'explication fournie pour justifier ce bilan. 


\section{Professeurs non-formés et non-accompagnés $(\mathrm{N}=9)$}

$$
\text { FR-B11, FR-A13) soit plus ou moins positifs (FR-B10, FR-A }
$$
troisième année de recherche. Un seul est négatif (FR-A11). Ceux qui sont positifs répondent que leur enseignement est plus centré sur les étudiants: "J'arrive à tenir compte du type d'étudiants à qui j'enseigne. » (CA-D10). Quant aux professeurs qui font un bilan plus ou moins positif, ils mettent généralement en cause le contexte dans lequel ils enseignent: "Ce n'est pas au niveau de mes méthodes que j'éprouve une difficulté; la difficulté réside principalement au niveau du système.» (FR-A10). Un professeur (FR-A14) précise que, de toute façon, l'enseignement n'est pas vraiment valorisé à l'université. FR-A11, pour sa part, déplore ne pas pouvoir choisir le type de cours qu'il aime donner.

\section{Professeurs accompagnés $(\mathrm{N}=7)$}

Tout comme pour ceux sans formation ni accompagnement, les professeurs accompagnés se partagent à peu près également entre ceux qui sont positifs (CA-D20, CA-D21, FR-B21, FR-B22) et ceux qui sont plus ou moins positifs (FR-B20, FR-A20, FRA21). Les premiers expliquent travailler à développer leurs stratégies d'enseignement pour se centrer davantage sur les étudiants, malgré le contexte dans lequel ils exercent. Les autres plus ou moins positifs manifestent leur volonté de poursuivre leurs efforts pour améliorer leur enseignement : « On apprend de ses erreurs chaque année. » (FRB20).

\section{Professeurs formés $(\mathrm{N}=10)$}

À l'exception de deux professeurs (FR-C51 négatif et CA-D51 plus ou moins positif), les professeurs formés ont expliqué avoir maintenu une vision positive au cours des années ou avoir progressé positivement, car ils parvenaient à se centrer davantage sur les étudiants ou à mieux les comprendre : «Je sens que les exercices sont plus clairs et que les étudiants voient l'utilité du cours. » (CA-D50); «Je connais mieux les étudiants, ils semblent plus contents de leurs apprentissages. » (CA-D54).

\section{Professeurs formés et accompagnés $(\mathrm{N}=8)$}

La moitié des professeurs formés et accompagnés font un bilan positif de leur expérience (CA-E34, CA-E31, CA-E32, FR-A32). Leurs commentaires concernent principalement leur enseignement : «Je prends des notes après chaque séance sur ce que je souhaite modifier, améliorer.»(CA-E34). Les quatre autres professeurs (CA-E33, CA-E30, FR-A30, FR-A31) plus ou moins positifs expliquent qu'ils sont limités dans leur démarche à cause, par exemple, du manque de ressources concrètes pour retravailler leur cours ou du trop grand nombre d'étudiants en cours.

\subsection{Commentaires des enseignants sur les ressources mobilisées pour leur développement pédagogique}

Dans cette section, nous détaillerons les réponses données par les professeurs à l'an 3 au sujet des autres ressources qu'ils ont mobilisées pour se former ou s'informer au 
cours de la recherche. À cette occasion, certains professeurs sont revenus sur l'utilité de la formation et de l'accompagnement offerts dans le cadre de la recherche. Les données concernant les ressources sont présentées de manière abrégée dans les tableaux l'annexe 2.

\section{Professeurs non formés, non accompagnés $(\mathrm{N}=9)$}

50 La grande majorité des professeurs sans formation ni accompagnement disent se former, s'informer surtout en échangeant avec des pairs, et ce afin d'obtenir des conseils, poser des questions concernant leurs pratiques pédagogiques, dont notamment la gestion de classe, et découvrir de nouvelles pratiques comme l'utilisation de l'apprentissage par problèmes (APP). Toutefois, deux professeurs (FR-A12, FR-A14) rapportent que les échanges ont souvent peu de valeur sur le plan pédagogique, car ils demeurent superficiels. Un seul (CA-D11) répond n'avoir eu aucun échange avec ses pairs.

Quelques professeurs affirment avoir aussi participé à de courts ateliers thématiques (CA-D11, FR-A11, FR-B11) ou consulté des ouvrages (FR-A10, FR-B11). Un professeur (FR-A11) dit avoir ainsi découvert des outils technologiques, mais qui ne sont pas nécessairement intéressants pour l'apprentissage. Pour un autre (CA-D11), ces rencontres ne sont pas très fructueuses, car les professeurs finissent souvent par discuter de leurs expériences personnelles. Ce dernier dit préférer suivre ses intuitions personnelles. Un professeur (FR-A13) n'a effectué aucune démarche pour se former ou s'informer par manque de temps et d'intérêt.

\section{Professeurs accompagnés $(\mathrm{N}=7)$}

Parmi les professeurs accompagnés, cinq ont parlé de l'accompagnement (sauf M 201 et FR-B22). Quatre (CA-D21, FR-B20, FR-A20, FR-A21) mentionnent qu'il les a aidés à développer leurs pratiques en les poussant à réfléchir ou à utiliser de nouvelles approches pour enseigner et évaluer. Un professeur (FR-B21) mentionne qu'il n'a pas été utile, car rien n'a fonctionné.

Plusieurs professeurs (CA-D20, CA-D21, FR-B22, FR-A20, FR-A21) expliquent les bienfaits d'échanger avec les pairs. Certains répondent avoir également suivi des ateliers (CAD21, FR-B20, FR-B22), un seul (FR-B22) aura consulté un ouvrage. Parmi ceux-ci, un professeur (CA-D21) répond que les ateliers ne l'ont pas aidé alors qu'un autre dit y avoir découvert l'APP qui lui a permis de rendre ses cours plus dynamiques et intéressants (FR-B22).

\section{Professeurs formés $(\mathrm{N}=10)$}

Un seul professeur formé (FR-A50) mentionne que la formation lui a permis de développer des outils et des pratiques pédagogiques. La grande majorité des professeurs (CA-D51, CA-D50, CA-D52, CA-D53, CA-D54, CA-D55, CA-D56, FR-A50, FR-C50) met l'accent sur les échanges qu'ils ont eus avec leurs pairs durant la formation au sujet de la gestion de classe, des différentes façons d'impliquer les étudiants, des stratégies pédagogiques, de l'évaluation et de l'utilisation d'outils. Un des professeurs dit cependant préférer échanger avec ses pairs, car c'est moins formel et plus efficace (CAD51). Cinq professeurs (CA-D50, CA-D52, CA-D53, CA-D55 et FR-C50) ont suivi des 
ateliers d'une ou deux heures qui leur ont permis de réfléchir à leurs pratiques et d'apprendre à communiquer. Trois (CA-D50, CA-D52, CA-D53) auront occasionnellement consulté un ouvrage afin de se renseigner sur l'utilisation des diapositives et sur l'apprentissage actif. Un professeur (FR-C51) n'aura eu recours à aucune autre ressource en dehors de la formation suivie.

\section{Professeurs formés et accompagnés $(\mathrm{N}=8)$}

Seuls les professeurs canadiens (CA-E30, CA-E31, CA-E32, CA-E33, CA-E34) indiquent que la formation et l'accompagnement ont été utiles. Ils rapportent qu'ils leur ont donné certaines bases théoriques, les a aidés à planifier leurs cours, diversifier leurs stratégies, améliorer leur capacité à communiquer avec les étudiants, évaluer les apprentissages ou gérer leur classe. Ils disent aussi avoir échangé avec les pairs, mais seul CA-E32 spécifie en avoir tiré des bénéfices. Deux professeurs (CA-E32 et CA-E33) ont suivi plusieurs ateliers courts qu'ils disent avoir appréciés. CA-E33 considère avoir beaucoup appris par lui-même. Les Canadiens n'auront pas consulté d'ouvrages.

Les trois professeurs français (FR-A30, FR-A31, FR-A32) n'ont pas eu le même engouement que les professeurs canadiens pour la formation et l'accompagnement. FRA30 dit avoir beaucoup échangé au sujet des grilles d'évaluation sans avoir l'intention de les utiliser. Un professeur (FR-A31) indique qu'il avait déjà fait beaucoup de travaux sur la pédagogie. FR-A32 explique avoir choisi la formation et l'accompagnement par curiosité, pour avoir des conseils. Il ajoute qu'en fait la pédagogie c'est « quelque chose de logique ». Aucun Français n'a suivi un atelier ou consulté un ouvrage.

\section{Discussion sur le développement des pratiques centrées sur l'apprentissage}

Dans la section qui suit, nous discuterons les résultats en fonction des trois objectifs de la recherche : 1) déterminer le niveau de centration sur l'apprentissage des pratiques d'enseignement des nouveaux professeurs au cours de trois années consécutives selon les différents dispositifs utilisés; 2) décrire les perceptions de chaque professeur à l'égard de l'évolution de ses pratiques; 3 ) circonscrire les autres ressources utilisées par ces professeurs pour développer leur pratiques et en préciser l'utilité.

\subsection{Le niveau de centration des pratiques observées selon les dispositifs mis en place}

L'analyse des résultats agrégés présentés dans la section 4.1, nous amène à faire un certain nombre de commentaires. Dans un premier temps, notons que le niveau de centration sur l'apprentissage des professeurs appartenant aux différents regroupements ne se distinguent pas selon leur discipline. Ce résultat correspond à celui de Stes et al. (2010) voulant que les dispositifs regroupant des professeurs de disciplines diverses ont des effets comparables. Par ailleurs, nous retrouvons dans tous les regroupements quelques professeurs ayant des pratiques qui progressent et qui régressent. Les professeurs dont le niveau de centration progresse ont, dans 6 cas sur 8 , une valeur initiale inférieure à 1,2 , alors que dans les cas où les pratiques régressent, elle est supérieure ou égale à 2 dans les mêmes proportions. Il apparaît plus facile de 
progresser si le niveau de départ est faible que si la pratique est déjà bien centrée sur les apprentissages. Les dispositifs mis en place ne semblent pas responsables de ces progressions ou régressions, car le même phénomène se retrouve chez les non formés non accompagnés. De fait, les quatre regroupements se ressemblent quant à la variété des évolutions individuelles observées. On peut présumer ici que les facteurs personnels et institutionnels influencent tout autant le cheminement des professeurs que les dispositifs offerts.

Les résultats de l'annexe 2 font également ressortir que les pratiques des professeurs canadiens progressent plus que celles des français. Une partie de l'explication pourrait être que les Canadiens progressent à partir d'un niveau initial moyen moins élevé alors que les Français ont du mal à conserver leur niveau initial moyen plus élevé. Un autre facteur possible apparaît lorsqu'on regarde l'université de rattachement : six des huit professeurs concernés viennent de la même université. Le contexte institutionnel semble ici plus déterminant que le contexte national. Il serait donc intéressant dans une recherche ultérieure d'interroger des facteurs institutionnels comme le soutien à l'enseignement, la liberté de choix des méthodes pédagogiques, la valorisation de l'engagement pédagogique, l'équilibre entre activités de recherche et d'enseignement, pour mieux expliquer les différences entre les sujets. Par ailleurs, aucune tendance n'apparaît concernant les champs disciplinaires dans lesquels les professeurs enseignent. Contrairement aux résultats de Kiffer (2016), les spécificités épistémologiques de chaque discipline n'expliquent pas les résultats.

Considérant les données de l'annexe 3, nous observons que tous les professeurs ont adopté des pratiques émanant de l'approche cognitive, bien que celles-ci soient plus fréquemment de niveau 1, moins souvent de niveau 2 et nettement moins fréquemment de niveau 3. Ce qui veut dire que les professeurs demeurent plus souvent au contrôle des opérations cognitives et métacognitives ou qu'ils posent eux-mêmes des questions pour amener les étudiants à effectuer des activités simples. Saint-Pierre et al. (2014) observaient aussi, chez des professeurs œuvrant pourtant dans des programmes innovants, un retour à des pratiques de premier niveau après quelques années de pratiques cognitives complexes. Ils l'expliquaient de la manière suivante: "L'enseignant négocie sa manière d'intervenir selon le contexte, mais aussi selon ses préférences individuelles. Il arrive donc que les préférences de certains universitaires, experts de leur domaine, se manifestent par la transmission de leur expertise. » (SaintPierre et al., 2012, p.12). En fait, comme le constataient Romainville et Michaud (2012) ainsi que Ménard (2012), les professeurs ont tendance à se rapprocher des pratiques qu'ils ont connues comme étudiants, bien qu'ils semblent avoir tous été sensibilisés aux besoins de formation des étudiants d'aujourd'hui.

61 Si nous détaillons maintenant les différentes catégories de pratiques, "Susciter et exploiter les interactions » est systématiquement en progression au niveau 1 dans tous les groupes. Nous retrouvons dans les témoignages des professeurs présentés dans la section suivante cet intérêt marqué pour l'interactivité. En revanche, la catégorie " Donner du sens à l'apprentissage ", bien qu'elle ait obtenu un score plutôt élevé à l'an 1 , est en recul notamment pour le groupe des non formés non accompagnés. Pourtant, la motivation est considérée déterminante pour soutenir la démarche d'apprentissage (Viau, 2014). Peut-être qu'avec les années d'enseignement, l'utilité, l'intérêt ou l'importance des savoirs enseignés devenant plus évidents pour les professeurs, ils oublient de l'expliciter aux étudiants. Par ailleurs, les pratiques cognitives complexes 
qui permettent l'intégration des apprentissages ("Intégrer l'évaluation dans les situations d'apprentissage » et "Favoriser le transfert des nouveaux apprentissages ») sont moins souvent mises en œuvre que les autres catégories.

En somme, il apparaît que c'est davantage le niveau de centration sur l'apprentissage des professeurs en début de carrière de même que le contexte dans lequel ils exercent qui déterminent l'évolution des pratiques que les dispositifs mis en place au cours des années. Par ailleurs, il faut mettre en exergue le fait que les professeurs ont déjà tous adopté des pratiques cognitives, mais qu'elles gagneraient à évoluer vers un plus grand engagement des étudiants dans leurs apprentissages (Annoot, 2014) et vers la mise en place d'activités de résolution de problèmes et de transfert (Frenay et Bédard, 2004; Tardif, 1999).

\subsection{Les pratiques perçues par les professeurs}

63 Voyons maintenant de quelle manière les professeurs perçoivent leurs pratiques à l'an 3 de la recherche. D'emblée, le fait que les formés ont des perceptions majoritairement positives alors que les autres regroupements de professeurs ont des perceptions positives, plus ou moins positives ou négatives, questionne. La formation courte seule donnerait-elle davantage confiance en soi? Il ne faut pas oublier que les formés sont ceux qui ont eu le plus souvent recours à d'autres sources de formation et d'information. Il n'y a donc plus seulement la formation courte qui est en cause.

Il est intéressant ici de noter que la plupart des professeurs font un bilan qui s'adosse aux pratiques observées en ce qui concerne la qualité de leurs pratiques. Seulement deux professeurs dont les pratiques accusent un recul notable ne semblent pas les percevoir en régression. Ce bilan fait par les professeurs confirme l'intérêt de les interroger sur leurs pratiques comme l'ont fait Bailly et al. (2015), car ils semblent conscients de leur situation.

Un autre résultat à mettre en relief est que la plupart des professeurs font un bilan de leurs pratiques en démontrant un intérêt certain pour l'apprentissage et pour leurs étudiants. Ainsi, les professeurs qui font un bilan positif l'expriment clairement. Quant aux accompagnés plus ou moins positifs, ils affirment leur volonté de poursuivre leurs efforts dans ce sens alors que les formés accompagnés ainsi que les non formés accompagnés plus ou moins positifs et négatifs expliquent faire face à des situations qui les limitent ou à un contexte qui ne favorisent pas un changement de pratiques. Nous notons que les disciplines des professeurs de ces deux derniers groupes sont variées, mais que plusieurs d'entre eux proviennent de la même université. Encore une fois, le contexte de la pratique d'un milieu universitaire semble expliquer la situation.

L'intérêt manifesté par les professeurs se traduit par des pratiques qu'ils décrivent comme tenir compte de ses étudiants, répondre à chaque question, changer d'exemples, essayer de rendre son cours plus interactif, faire participer les étudiants et tenter d'implanter des nouveautés. Les professeurs décrivent ici des pratiques dont ils sont souvent le principal acteur (premier niveau de centration) et ils mettent l'accent sur l'interactivité comme nous l'avions observé.

67 Les résultats nous permettent d'affirmer que les professeurs peuvent rendre compte de leur situation. Ils permettent aussi de savoir que la plupart des nouveaux professeurs manifestant de l'intérêt pour l'apprentissage ont adopté des pratiques proposées par les cognitivistes qu'ils soient formés, accompagnés ou pas. Ils ont donc été rejoints par 
le discours actuel en pédagogie universitaire. Toutefois les pratiques adoptées demeurent basiques et mériteraient d'évoluer vers les niveaux de centration 2 et 3 . Finalement, comme le soutiennent Beguin (2007) ainsi que Dall'Alba et Sandberg (2006), le contexte universitaire paraît influencer les pratiques d'enseignement de certains professeurs.

\subsection{Autres ressources consultées} particulièrement utiles. La recherche de Kiffer (2016) indique aussi que cette ressource est considérée comme importante et utile aux yeux des nouveaux professeurs. Il faut noter que le contenu, la fréquence et la pertinence de ces échanges informels nous échappent complètement, contrairement au contenu de la formation et de l'accompagnement.

71 Les ateliers thématiques d'une ou deux heures sont la deuxième ressource la plus exploitée, ils semblent répondre à des besoins spécifiques. Les formés qui les fréquentent le plus, y voient peut-être le prolongement logique de leur formation alors que les non formés non accompagnés qui les fréquentent beaucoup moins, sont critiques quant à leur pertinence. La consultation d'ouvrages est nettement plus rare.

\section{Conclusion}

Cette recherche s'est intéressée aux effets de la formation ou de l'accompagnement sur les pratiques observées, aux perceptions des nouveaux professeurs canadiens et français concernant leurs pratiques ainsi qu'aux autres ressources exploitées par eux pour se former ou s'informer au fil du temps. 34 nouveaux professeurs formés, accompagnés, formés et accompagnés et sans formation ni accompagnement ont été 
suivis durant trois ans. En ce qui concerne les pratiques pédagogiques observées, l'analyse démontre qu'au-delà du fait que des professeurs ont progressé ou régressé à l'intérieur de chaque groupe, la majorité ne développe pas son niveau de centration sur l'apprentissage au cours des trois années de la recherche.

73 En détaillant les différentes catégories de pratiques de chacun, il apparaît que les professeurs ont tous adopté des pratiques cognitives dès leur entrée en carrière. La catégorie qui progresse le plus souvent est «Favoriser et exploiter les interactions». Cet intérêt pour l'interactivité est également bien présent lorsque les professeurs font le bilan de leurs pratiques au terme de la recherche.

74 À savoir quelles autres ressources les professeurs ont le plus souvent utilisées pour se former ou s'informer au cours des trois années de la recherche? Il apparait que les échanges avec les pairs constituent le moyen le plus fréquemment exploité et que les formés sont ceux qui ont utilisé le plus fréquemment d'autres ressources.

75 Les résultats de cette recherche démontrent qu'une formation courte ou quelques séances d'accompagnement n'ont pas eu d'effets marqués sur l'évolution des niveaux de centration observés, que les pratiques des professeurs sont le plus souvent de niveau 1, que la discipline d'enseignement des professeurs ne permet à aucun moment d'appréhender les résultats, mais que l'appartenance à un même contexte universitaire explique peut-être le fait que plusieurs professeurs ont régressé au fil du temps. Les résultats mettent aussi en relief que la plupart des professeurs s'intéressent à l'apprentissage étudiant, qu'ils ont adopté des pratiques correspondant à l'approche cognitive et qu'ils ont senti le besoin de recourir à d'autres sources de formation et d'information au cours des années.

76 Cette étude démontre aussi la complexité de la recherche voulant documenter le développement pédagogique. Il apparait en effet impossible d'affirmer qu'un dispositif produit en soi un effet sur les pratiques des professeurs, sans prendre en compte les autres dispositifs utilisés et sans aucun doute les motivations des personnes et le contexte de la pratique. Il faut y ajouter l'expérience d'enseigner qui joue certainement un rôle dans le développement pédagogique d'un professeur, soit en le soutenant, soit en l'amenant à effectuer un retour sur ses préférences de départ comme le soutiennent Saint-Pierre et al.(2012). En fait, tout cela relativise les résultats des recherches qui disent examiner les effets d'un dispositif sur le développement pédagogique des professeurs.

77 Nous retenons, par ailleurs, que pour intervenir de manière plus efficace en pédagogie universitaire, différentes pistes mériteraient d'être explorées. Ainsi, on devrait se demander de quelle manière les formations offertes pourraient avoir un effet plus marqué sur les niveaux de centration sur l'apprentissage, notamment les niveaux 2 et 3? Il semble que les formations comme celles qui ont été dispensées ont un effet sur les perceptions des professeurs, mais peu sur les pratiques. Comme l'expliquent Frenay et al. (2010), la formation doit donner le temps aux professeurs de réfléchir, de se questionner et d'expérimenter d'autres pratiques. Cela prend du temps et une formation courte ne permet pas un réel changement de cap. De même, il serait pertinent de comparer différentes modalités d'accompagnement en milieu universitaire en dépassant les référentiels les plus connus en pédagogie universitaire comme, par exemple, ceux de Paul (2015), de Kolb (1984) ou de Schön (1983). Il serait également intéressant d'examiner de plus près les effets des autres ressources au développement des professeurs, particulièrement les échanges avec les pairs qui se font 
souvent de manière ponctuelle et informelle, de même que la contribution cumulative des autres ressources.

Finalement, il serait souhaitable de se poser des questions sur le contexte dans lequel exercent les professeurs. Une université qui met en place un service pédagogique devrait veiller à offrir des conditions propices au changement, comme valoriser l'enseignement dans le cadre universitaire et une certaine liberté de choix sur le plan pédagogique. La question se pose: quel service est mis en place pour quels changements?

Pour terminer, il est nécessaire de mentionner que cette recherche comporte des limites par la diversité même de ses sujets, le nombre d'observations réalisées et le déséquilibre entre le nombre de professeurs représentés dans les universités participantes. Ainsi, le fait que les Français et les Canadiens ne soient pas en nombre équivalent dans chaque groupe a certainement produit des résultats entre les groupes qui demeurent délicats à comparer. De même, étant donné le nombre limité d'observations, il est difficile de prétendre que ce qui a été observé est représentatif de l'ensemble des pratiques d'un professeur. Toutefois, le bilan que font les professeurs de leurs pratiques au terme des trois de recherche correspondant la plupart du temps aux observations effectuées, nous permet tout de même de penser que cette recherche a produit des résultats qui valent la peine d'être pris en considération et qui mérite d'être reproduite dans des contextes d'exercice variés.

\section{BIBLIOGRAPHY}

Annoot, E. (2014). De l'accompagnement à la pédagogie universitaire : quels enjeux pour la formation des enseignants-chercheurs? Recherche et formation, 77, 17-28. https:// journals.openedition.org/rechercheformation/2298

Arpin, L. et Capra. L. (2008). Accompagner l'enseignant dans son parcours professionnel. Montréal, Canada : Chenelière Éducation.

Bailly, B., Demougeot-Lebel, J. et Lison, C. (2015). La formation d'enseignants universitaires nouvellement recrutés : quelles retombées? Revue internationale de pédagogie de l'enseignement supérieur, 31(3). http//ripes.revues.org/pdf/990

Beguin, P. (2007). Prendre en compte l'activité de travail pour concevoir. Activités, 4(2), 107-114.

Bertrand C. (2014). Soutenir la transformation pédagogique dans l'enseignement supérieur. Paris, France : Ministère de l'Enseignement Supérieur et de la Recherche.

Biemar, S. (2012). Accompagner un groupe d'enseignants dans une école : une grille de compétences. Dans B. Charlier et S. Biémar (dir.), Accompagner : un agir professionnel (p. 17-33). Bruxelles, Belgique : De Boeck.

Clanet, J. et Talbot, L. (2012). De l'analyse des pratiques enseignantes à la mise à jour des compétences professionnelles : Vers plus d'efficacité? Phronesis, 1(3), 1-3. 
Clement, M., Di Napoli, R., Annelies Gilis, A., Buelens, H. et Frenay, M. (2011). Educational consultation for reflective-dialogic partnerships : a possible model. Recherche et Formation, 67, 31-47. https://journals.openedition.org/rechercheformation/1371

Conseil supérieur de l'Éducation. (2013). Avis au ministre de l'Enseignement Supérieur, de la Recherche, de la Science et de la Technologie. Parce que les façons de réaliser un projet d'études universitaires ont changé... Québec, Canada : Gouvernement du Québec. https://www.cse.gouv.qc.Ca/fichiers/ documents/publications/Avis/50-0480.pdf

Daele, A. et Sylvestre, S. (2016). Introduction générale. Contexte Enjeux et Objectifs. Dans A. Daele et S. Sylvestre (dir.), Comment développer le conseil pédagogique dans l'enseignement supérieur (p. 13-20). Bruxelles, Belgique : De Boeck.

Dall'Alba, G. et Sandberg, J. (2006). Unveiling professional development : A critical review of stage models. Review of Educational Research,76(3), 383-412.

Duguet, A., Lambert-Le Mener, M. et Morlaix, S. (2016). Les déterminants de la réussite à l'université. Quels apports de la recherche en Éducation? Quelles perspectives de recherche? Spiral-E. Revue de recherches en éducation, Supplément électronique, 57, 31-53. https://doi.org/ 10.3406/spira.2016.1745

Finelli, C. J., Ott, M., Gottfried, A. C., Hershock, C., O'neal, C. et Kaplan, M. (2008). Utilizing Instructional Consultations to Enhance the Teaching Performance of Engineering Faculty. Journal of Engineering Education, 97(4), 397-411.

Frenay, M. et Bédard, D. (2004). Des dispositifs de formation universitaire s'inscrivant de la perspective d'un apprentissage et d'un enseignement contextualisés pour favoriser la construction de connaissances et leur transfert. Dans A. Presseau et M. Frenay (dir.), Le transfert des apprentissages (p. 239-267). Québec, Canada : Presses de l'université Laval.

Frenay, M., Jorro, A. et Poumay, M. (2011). Développement pédagogique, développement professionnel et accompagnement. Recherche et formation, 67, 105-116. www.inrp.fr/publications/ edition-electronique/recherche-et-formation/RR067-7.pdf

Frenay, M., Saroyan, A., Taylor, L., Bédard, D., Clement, M., Rege Colet, N., Paul, J.-J. et Kolmos, A. (2010). Accompagner le développement pédagogique des professeurs universitaires à l'aide d'un cadre conceptuel original. Revue française de pédagogie, 172, 63-76.

Hanbury, A., Prosser, M. et Rickinson, M. (2008). The differential impact of UK accredited teaching development programs on academics'approaches to teaching. Studies in Higher Education, 33(4), 449-483.

Jonnaert, P. et Vander Borght, C. (2009). Créer des conditions d'apprentissage : un cadre de référence socioconstructiviste pour une formation didactique des enseignants ( $3^{\mathrm{e}}$ éd.) Bruxelles, Belgique : De Boeck Université.

Kozanitis, A., Demougeot-Lebel, J. et Daele, A. (2016). Le soutien pédagogique individuel. Dans A. Daele et S. Sylvestre (dir.), Comment développer le conseil pédagogique dans l'enseignement supérieur (p. 24-40). Bruxelles, Belgique : De Boeck.

Kiffer, S. (2016). La construction des compétences d'enseignement des enseignants-chercheurs novices de l'université en France (Thèse de doctorat inédite). Université de Strasbourg, France.

Kolb, D. A. (1984). Experiential Learning - Experience as the source of learning and development. Englewoods Cliffs, NJ : Prentice-Hall.

Langevin, L., Grandtner, A.-M. et Ménard, L. (2008). La formation à l'enseignement des professeurs d'université. Revue des sciences de l'éducation, 34(3), 643-664. 
Lenoir, Y. (2012). Étudier la pratique d'enseignement dans sa complexité : une démarche multidimensionnelle. Dans J. Clénet, P. Maubant et D. Poisson (dir.), Formations et professionnalisations : à l'épreuve de la complexité (p. 229-256). Paris, France : L'Harmattan.

Luzeckyj, A. et Badger, L. (2008). Literature review for preparing academics to teach in higher education (PATHE). Australia : Australian Learning \& Teaching Council. https:// www.academia.edu/876814/ Literature_review_for_Preparing_Academics_to_Teach_in_Higher_Education_PATHE_

Ménard, L. (2012). Apprentissage en classe et persévérance au premier cycle universitaire. Dans M. Romainville et C. Michaud (dir.), Réussite, échec et abandon dans l'enseignement supérieur (p. 177-198). Bruxelles, Belgique : De Boeck.

Parmentier P. et Paquay, L. (2002). En quoi les situations d'enseignement/apprentissage favorisent-elles la construction de compétences? Développement d'un outil d'analyse : le Comp.A.S. Louvain-la-Neuve, Belgique : UCL.

Paul, M. (2015). L'accompagnement de la notion au concept. Éducation permanente, 4(205), 21-30.

Postareff, L., Lindblom-Ylänne, S. et Nevgi, A. (2008). A follow-up study of the effect of pedagogical training on teaching in higher education. Higher Education, 56, 29-43.

Raby, G. (2011). Pour une licence attractive doublement qualifiante, permettant une poursuite d'études en master et une insertion professionnelle, une licence assurant un parcours de réussite aux étudiants. Contribution écrite au colloque annuel de la Conférence des Présidents d'université Toulouse. http://inpact.inp-toulouse.fr/CPU2011/contributions \%20ecrites.html, consulté le 3 juin 2011.

Rivers, J. (2005). Academic staff development. A summary of a synthesis of research on the impact of academic staff development programmes on student outcomes in undergraduate tertiary study. New Zealand, Australia : Ministry of Education.

Rocheleau, J. (2009). Les théories cognitivistes de l'apprentissage. Université du Québec à TroisRivières, Canada. https://oraprdnt.uqtr.uquebec.Ca/pls/public/docs/GSC332/

F766183874_Approche_cognitiviste_apprentissage2009_10_05.pdf

Romainville, M. et Michaud, C. (2012). Réussite, échec et abandon dans l'enseignement supérieur. Bruxelles, Belgique : De Boeck.

Schön, D.-A. (1983). The reflective practitioner : how professionals think in action. New-York, USA : Basic books.

Saint-Pierre, L., Bédard, D. et Lefebvre, N. (2012). Enseigner dans un programme universitaire innovant : de nouveaux rôles à apprivoiser, des actes pédagogiques à diversifier. Canadian Journal for the Scholarship of Teaching and Learning, 3(1). https://ir.lib.uwo.ca/cgi/viewcontent.cgi? article $=1059 \&$ context $=$ cjsotl_rcacea

Saint-Pierre, L., Bédard, D. et Lefebvre, N. (2014). Une grille d'analyse de ses interventions en classe. Dans L. Ménard et L. Saint-Pierre (dir.), Se former à la pédagogie de l'enseignement supérieur (p. 35-52). Montréal, Canada : Association québécoise de pédagogie collégiale.

Stes, A., Min-Leliveld, M., Gijbels, D. et Van Petegem, P. (2010). The impact of instructional development in higher education : The state-of-the-art of the research. Educational Research Review, 5(1), 25-49. http://www.sciencedirect.com/science/article/pii/S1747938X09000359

Stes, A. et Van Petegem, P. (2011). La formation pédagogique des professeurs dans l'enseignement supérieur Une étude d'impact. Recherche et formation, 67, 15-30.

Tardif, J. (1997). Pour un enseignement stratégique ( $2^{\mathrm{e}}$ éd.). Montréal, Canada : Les Éditions Logiques. 
Tardif, J. (1999). Le transfert des apprentissages. Montréal, Canada : Les Éditions Logiques.

Taylor, L. K. et Rege Colet, N. (2010). Making the shift from faculty development to educational development : A conceptual framework grounded in practice. Dans A. Saroyan et M. Frenay (dir.), Building Teaching Capacities in Higher Education : A Comprehensive International Mode (p. 139-167). Sterling, VA : Stylus.

Trigwell, K., Caballero Rodriguez, K. et Han, F. (2012). Assessing the impact of a university teaching development program. Assessment and Evaluation in Higher Education, 37(4), 499-511.

Viau, R. (2014). Savoir motiver les étudiants. Dans L. Ménard et L. St-Pierre (dir.), Se former à la pédagogie de l'enseignement supérieur (p. 235-254). Montréal, Canada : Collection Performa.

\section{NOTES}

1. Ils constituent la clientèle prioritaire en France, car, depuis la rentrée 2018, le gouvernement a décrété que les maîtres de conférences nouvellement recrutés ont l'obligation de se former à la pédagogie au cours de leur première année d'exercice (Décret n 2017-854 ; mai 2017).

2. Cette recherche a été financée par le programme Savoir du Conseil de recherches en sciences humaines du Canada (CRSH).

3. Nous avons choisi l'« approche cognitive » comme cadre de référence (nous le définissons dans la section 2). Nous n'invoquons pas le constructivisme bien que le constructivisme et le cognitivisme soient très souvent associés. Comme l'affirment par exemple Jonnaert et Vander Borght (2009), il nous semble que les deux théories s'entendent certes sur le processus de construction des connaissances, mais qu'elles divergent quant aux moyens pour favoriser l'apprentissage.

\section{ABSTRACTS}

en

Many Canadian and French universities offer short-term teaching and counselling to support new teachers to focus more on student learning. But to what extent does training and counselling have an effect on the level of focus on student learning, whether or not they have benefited from these devices? This study presents data collected by observation and interview, over three years, from 34 new French and Canadian teachers, 9 of whom were untrained and unaccompanied, 7 unaccompanied, 10 trained and 8 trained and accompanied. With respect to the pedagogical practices observed, the analysis shows that the learning focus of each group did not change significantly and that most individuals in these groups maintain their three levels of learning focus over time. However, some of the various disciplines progress or regress significantly. Those who regress come mainly from the same university. Peer-to-peer exchanges appear to be the other most commonly used source of training and information. This research demonstrates the complexity of the educational development process. It seems to us impossible today to say that a device in itself has an effect on the practices of teachers, without taking into account the other devices used and, no doubt, the motivations of the people and the context of the practice. 
fr

Plusieurs universités canadiennes et françaises offrent une formation à l'enseignement de courte durée et un accompagnement pédagogique aux nouveaux professeurs afin de les amener à se centrer davantage sur l'apprentissage étudiant. Mais dans quelle mesure ces dispositifs ont-ils un effet sur le niveau de centration sur l'apprentissage des professeurs selon qu'ils aient bénéficié ou pas de ces dispositifs? Cette étude présente des données collectées par observation et par entrevue, durant trois années, auprès de 34 nouveaux professeurs français et canadiens dont 9 sans formation ni accompagnement, 7 uniquement accompagnés, 10 formés et 8 formés et accompagnés. En ce qui concerne les pratiques pédagogiques observées, l'analyse démontre que les degrés de centration sur l'apprentissage de chaque groupe n'ont pas évolué de façon marquée et que la plupart des individus de ces groupes maintiennent au fil du temps, leurs trois niveaux de centration. Toutefois quelques-uns, de disciplines variées progressent ou régressent de façon notable. Ceux qui régressent viennent majoritairement d'une même université. Il apparaît que les échanges avec les pairs constituent l'autre source de formation et d'information la plus fréquemment exploitée. Cette recherche démontre la complexité du processus de développement pédagogique. Il nous semble aujourd'hui impossible d'affirmer qu'un dispositif produit en soi un effet sur les pratiques des professeurs, sans prendre en compte les autres dispositifs utilisés et, sans aucun doute, les motivations des personnes et le contexte de la pratique.

INDEX

Mots-clés: formation, accompagnement pédagogique, nouveaux professeurs, pratiques d'enseignement, université

\section{AUTHORS}

\section{LOUISE MÉNARD}

Université du Québec à Montréal, menard.louise@uqam.ca

\section{CHRISTIAN HOFFMANN}

Université Grenoble Alpes, christian.hoffmann@neel.cnrs.fr

\section{SÉBASTIEN BOUCHER}

Université du Québec à Montréal, sebb.boucher@gmail.com

MARTIN RIOPEL

Université du Québec à Montréal, riopel.martin@uqam.ca 